Note

\section{A Kinetic Model for Limestone Decomposition under BOF Steelmaking Condition}

\author{
Lei SHAO, Shan YU, Biao TANG, Xiao-ming WANG and \\ Zong-shu ZOU*
}

Key Laboratory of Ecological Utilization of Multi-metal Intergrown Ores of Education of Ministry, Northeastern University, Shenyang, 110819 P. R. China.

(Received on July 17, 2015; accepted on October 20, 2015; J-STAGE Advance published date: December 3, 2015)

In BOF steelmaking process, high-activity lime has been widely used to form an appropriate slag as a medium for the removal of non-volatile impurities from the hot metal treated. In recent years, however, great attention has been paid to the possibility of directly using limestone instead of lime in the furnace. Despite laboratory experiments and industrial trials have proven the feasibility of the technique, a list of underlying issues still require more considerations, e.g., thermodynamics and kinetics of steelmaking reactions and limestone evolution during blowing under the new condition. The main motivation behind this paper is to establish a kinetic model for limestone decomposition under BOF steelmaking condition on the basis of shrinking core hypothesis. The Kozeny-Carman equation that correlates pressure gradient with the corresponding velocity is integrated into the model in order to account for the $\mathrm{CO}_{2}$ flow through the lime layer packed with small grains. The model is demonstrated using a set of experimental data and is finally verified by comparing the obtained thermal conductivity and grain size with available values in the literature.

KEY WORDS: limestone decomposition; BOF steelmaking; macrokinetics; pressure gradient driven flow; greenhouse gas emission.

\section{Introduction}

In BOF steelmaking process, high-activity lime has been widely used to form an appropriate slag as a medium for the removal of non-volatile impurities from the hot metal treated. ${ }^{1,2)}$ In recent years, however, great attention has been paid to the possibility of directly using limestone instead of lime in the furnace. The main reason for such interest is that the implementation of the above technique will be of appreciable benefit, both from environmental and economic points of view. In the conventional route, limestone is calcined in a shaft or rotary kiln at a temperature of around $1273 \mathrm{~K}$ and after cooling down, the produced lime is transported and then charged into a converter. ${ }^{3)}$ The problem of the route, among others, lies in that the sensible heat embodied by the high-temperature lime and its by-product $\mathrm{CO}_{2}$ can barely be utilized and moreover, a big portion of the $\mathrm{CO}_{2}$ is emitted into the environment. By contrast, the direct use of limestone can readily avoid such energy waste and greenhouse gas emission. The intensive endothermic reaction of limestone decomposition plays an

\footnotetext{
* Corresponding author: E-mail: zouzs@mail.neu.edu.cn DOI: http://dx.doi.org/10.2355/isijinternational.ISIJINT-2015-417
}

important role as a coolant in adjusting the temperature of liquid bath, where the generated $\mathrm{CO}_{2}$ can also oxidize some impurity elements. ${ }^{4}$ ) As a result, the amount of scrap and oxygen consumed decreases, leading to a lower production cost. In addition, the formation of numerous $\mathrm{CO}_{2}$ bubbles will promote local stirring and thus facilitate steelmaking reactions. 5 )

Despite laboratory experiments and industrial trials have proven the feasibility of the technique, a list of underlying issues still require more considerations, e.g., thermodynamics and kinetics of steelmaking reactions and limestone evolution during blowing under the new condition. As for limestone decomposition in a shaft or rotary kiln, the wellknown shrinking core or unreacted core model ${ }^{6,7)}$ can be employed in conjunction with measured conversion-time data to evaluate the controlling step and then to optimize the operation. The aforementioned model usually assumes that the temperature at the reaction front is around $1173 \mathrm{~K}$ (e.g., the decomposition temperature of limestone at $1 \mathrm{~atm}$ ) and driven by concentration gradient, the produced $\mathrm{CO}_{2}$ diffuses outwards through the porous lime blanket surrounding the internal core. The authors of the present study have attempted to adopt such a model to investigate the decomposition behavior of limestone in hot metal and molten slag at different temperatures ranging from $1523 \mathrm{~K}$ to $1623 \mathrm{~K}$, which correspond to the condition at the early stage of BOF steelmaking. ${ }^{8)}$ The obtained effective diffusivities of $\mathrm{CO}_{2}$ are commonly much higher than the theoretical ones, indicating that the model cannot mirror the actual process. In considering the higher temperature during BOF process, the temperature at the reaction front could rise above $1173 \mathrm{~K}$, causing an exponential increase in the decomposition pressure. Driven by high pressure gradient, macroscopic flow of $\mathrm{CO}_{2}$ through the porous layer can occur and therefore, the governing mechanism of $\mathrm{CO}_{2}$ transfer would switch from diffusion to fluid flow.

The main motivation behind this paper is to establish a kinetic model for limestone decomposition under BOF steelmaking condition on the basis of shrinking core hypothesis. The Kozeny-Carman equation that correlates pressure gradient with the corresponding velocity is integrated into the model in order to account for the $\mathrm{CO}_{2}$ flow through the lime layer packed with small grains. The model is demonstrated using a set of experimental data and is finally verified by comparing the obtained thermal conductivity and grain size with available values in the literature.

\section{Model Description}

In the previous work, cylindrical specimens with a diameter of $14 \mathrm{~mm}$ were immersed in hot metal or molten slag at specified temperatures for a certain time. The samples were then sliced and clear interfaces between calcined lime and unreacted limestone were observed, suggesting that the shrinking core hypothesis is still applicable for the liquidsolid system.

\subsection{Assumptions and Simplifications}

From a theoretical standpoint and as shown in Fig. 1, the following five steps accompanying the decomposition process take place in succession and interact with one another.

Step 1: Convective heat transfer through the film surrounding the solid to its surface.

Step 2: Conductive heat transfer through the calcined layer to the surface of the unreacted core.

Step 3: Decomposition reaction driven by the deviation of $\mathrm{CO}_{2}$ partial pressure from the equilibrium at the reaction front.

Step 4: Convective mass transfer of $\mathrm{CO}_{2}$ by fluid flow through the calcined layer.

Step 5: Mass transfer of $\mathrm{CO}_{2}$ through the surrounding film to the bulk liquid.

The measured conversion curves in the previous work 


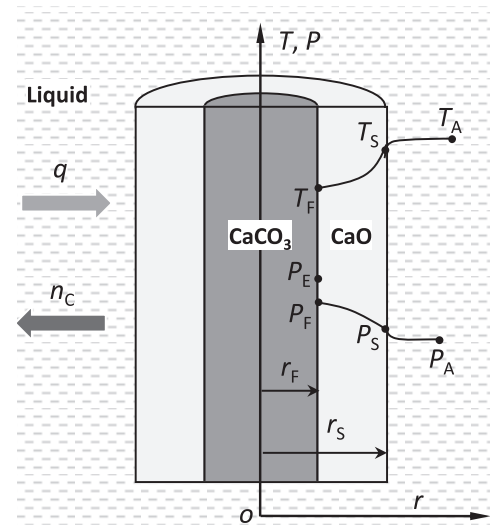

Fig. 1. Schematic representation of the decomposition process for a cylindrical specimen.

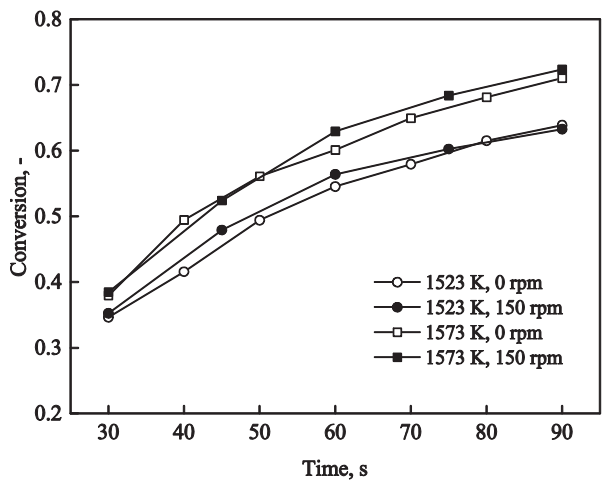

Fig. 2. Conversion curves at different rotating speeds and temperatures in hot metal bath.

have shown that the rotating speed of specimen only plays a minor role in controlling the decomposition process. For instance, the conversion curves at different rotating speeds and temperatures in hot metal bath are depicted in Fig. 2, where the conversion obviously rises with an increase in the temperature. However, it only varies slightly even as the rotating speed jumps to $150 \mathrm{rpm}$. The observation confirms that velocity and thus temperature boundary films, if still existent, are very thin at the solid surface. Therefore, Steps 1 and 5 are neglected in the current model, i.e., $P_{\mathrm{S}}=P_{\mathrm{A}}$ and $T_{\mathrm{S}}=T_{\mathrm{A}}$ (cf. Fig. 1). It is also worth noting that the following derivation of kinetic expressions is focused on a cylindrical object only because cylindrical specimens were employed in the experiments. Nevertheless, the derivation for spherical and plate objects are straightforward.

\subsection{Model Formulae}

Assuming a pseudo steady state and constant material properties, the heat flux by conduction in the lime layer is written as

$$
q=\lambda \frac{T_{\mathrm{A}}-T_{\mathrm{F}}}{r_{\mathrm{F}} \ln \frac{r_{\mathrm{S}}}{r_{\mathrm{F}}}}
$$

where $\lambda, T$ and $r$ are the effective thermal conductivity, temperature and position coordinate of radius. The subscripts $\mathrm{A}, \mathrm{F}$ and $\mathrm{S}$ denote ambient, reaction front and surface, respectively.

For the reaction at the front, the mass flux of $\mathrm{CO}_{2}$ is

$$
n_{\mathrm{C}, \mathrm{F}}=\frac{N_{\mathrm{C}, \mathrm{F}}}{2 \pi r_{\mathrm{F}}}=k \frac{P_{\mathrm{E}}-P_{\mathrm{F}}}{M_{\mathrm{C}} R T_{\mathrm{F}}}
$$

where $N, P, R$ and $M$ are the mass flow rate, pressure, gas constant and molar mass, respectively. The subscripts $\mathrm{C}$ and $\mathrm{E}$ stand for $\mathrm{CO}_{2}$ and equilibrium.
In Eq. (2), $k$ is the first-order reversible reaction rate constant

$$
k=k_{+}\left(1+\frac{1}{K}\right)
$$

where $k_{+}$and $K$ are the forward reaction rate constant and equilibrium constant. by $^{9)}$

The equilibrium pressure is described thermodynamically

$$
P_{\mathrm{E}}=4.1 \times 10^{12} \exp \left(-\frac{\Delta H}{R T_{\mathrm{F}}}\right)
$$

where $\Delta H$ is the reaction enthalpy of limestone decomposition.

For the $\mathrm{CO}_{2}$ flow driven by pressure gradient, the Kozeny-Carman equation is employed and its differential form is written as

$$
\frac{d P}{d r}=-180 \eta \frac{(1-\varepsilon)^{2}}{d^{2} \varepsilon^{3}} v
$$

where $\eta, \varepsilon, d$ and $v$ are the kinematic viscosity of $\mathrm{CO}_{2}$, average porosity and grain size of the lime layer, and fluid velocity, respectively.

On the basis of mass conservation, Eq. (6) gives the relationship between fluid velocity and mass flow rate,

$$
v=\frac{N_{\mathrm{L}, \mathrm{C}}}{\rho 2 \pi r} .
$$

where $N_{\mathrm{L}, \mathrm{C}}$ and $\rho$ are the mass flow rate of $\mathrm{CO}_{2}$ through the layer and fluid density.

Combining Eqs. (5) and (6) followed by integration, it reaches,

$$
P_{\mathrm{F}}-P_{\mathrm{S}}=A \frac{N_{\mathrm{L}, \mathrm{C}}}{2 \pi} \ln \frac{r_{\mathrm{S}}}{r_{\mathrm{F}}}
$$

where $A=180 \mu \frac{(1-\varepsilon)^{2}}{d^{2} \varepsilon^{3}}$ and $\mu$ is the dynamic viscosity.

Rearranging Eq. (7) gives

$$
n_{\mathrm{L}, \mathrm{C}}=\frac{N_{\mathrm{L}, \mathrm{C}}}{2 \pi r_{\mathrm{F}}}=\frac{P_{\mathrm{F}}-P_{\mathrm{A}}}{A r_{\mathrm{F}} \ln \frac{r_{\mathrm{S}}}{r_{\mathrm{F}}}}
$$

Considering $n_{\mathrm{C}}=n_{\mathrm{L}, \mathrm{C}}=n_{\mathrm{F}, \mathrm{C}}$, Eq. (9) is obtained combining Eqs. (2) and (8) followed by rearrangement.

$$
n_{\mathrm{C}}=\frac{P_{\mathrm{E}}-P_{\mathrm{A}}}{\frac{M_{\mathrm{C}} R T_{\mathrm{F}}}{k}+A r_{\mathrm{F}} \ln \frac{r_{\mathrm{S}}}{r_{\mathrm{F}}}}
$$

The mass flux of $\mathrm{CO}_{2}$ can also be expressed as

$$
n_{\mathrm{C}}=-\kappa_{\mathrm{C}} \frac{d r_{\mathrm{F}}}{d t}
$$

where $\kappa_{\mathrm{C}}$ and $t$ are the concentration of $\mathrm{CO}_{2}$ in limestone and time.

The heat flux and mass flux are related by

$$
q=\frac{\Delta H}{M_{\mathrm{C}}} n_{\mathrm{C}}
$$

In addition, conversion is calculated according to

$$
X=1-\left(\frac{r_{\mathrm{F}}}{r_{\mathrm{S}}}\right)^{2}
$$

Two analytical expressions of the liquid-solid system can then be achieved using the above equations followed by integration. 
Table 1. Expressions of the form functions and resistance terms.

\begin{tabular}{cc}
\hline Symbol & Expression \\
\hline$F_{1}(X)$ & $(1-X) \ln (1-X)+X$ \\
$F_{2}(X)$ & $1-\sqrt{1-X}$ \\
$\delta_{1}$ & $\frac{\Delta H K_{\mathrm{C}}}{M_{\mathrm{C}}\left(T_{\mathrm{A}}-T_{\mathrm{F}}\right)} \frac{r_{\mathrm{S}}^{2}}{4 \lambda}$ \\
$\delta_{2}$ & $\frac{K_{\mathrm{C}} M_{\mathrm{C}} R r_{\mathrm{S}}}{P_{\mathrm{E}}-P_{\mathrm{A}}} \frac{T_{\mathrm{F}}}{k}$ \\
$\delta_{3}$ & $\frac{K_{\mathrm{C}} r_{\mathrm{S}}^{2}}{P_{\mathrm{E}}-P_{\mathrm{A}}} \frac{A}{4}$ \\
\hline
\end{tabular}

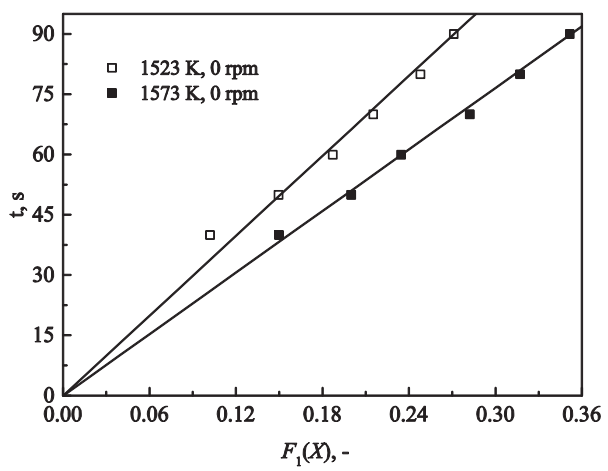

Fig. 3. Linearized decomposition diagram according to Eq. (13) at different temperatures in hot metal bath.

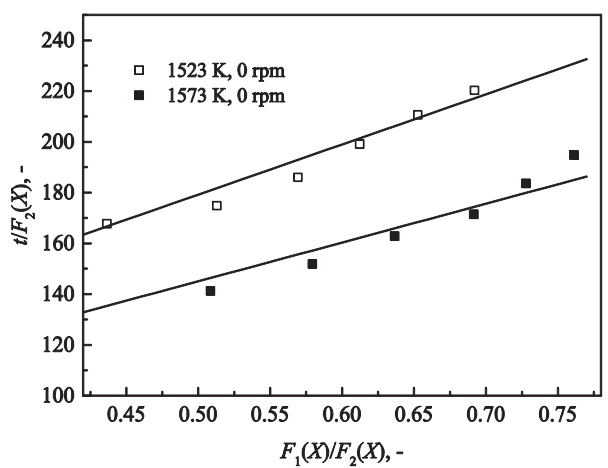

Fig. 4. Linearized decomposition diagram according to Eq. (15) at different temperatures in hot metal bath.

$$
\begin{array}{r}
t=\delta_{1} F_{1}(X) \ldots \ldots . \\
t=\delta_{2} F_{2}(X)+\delta_{3} F_{1}(X)
\end{array}
$$

On transformation, Eq. (14) becomes

$$
\frac{t}{F_{2}(X)}=\delta_{2}+\delta_{3} \frac{F_{1}(X)}{F_{2}(X)} \text {. }
$$

The form functions and resistance terms included in Eqs. (13) and (15) are summarized in Table 1.

\subsection{Model Demonstration and Validation}

Given experimentally measured data, the resistance terms containing model parameters can be easily obtained from the intercepts and slopes of the straight lines defined by Eqs. (13) and (15). For example, such diagrams are illustrated in Figs. 3 and $\mathbf{4}$ for different temperatures in hot metal bath. It is shown that the measured data can be approximated by
Table 2. Calculated results using the resistance terms

\begin{tabular}{ccc}
\hline Symbol & $1523 \mathrm{~K}$ & $1573 \mathrm{~K}$ \\
\hline$T_{\mathrm{F}}, \mathrm{K}$ & 1468 & 1486 \\
$\lambda, \mathrm{W} / \mathrm{m} \cdot \mathrm{K}$ & 1.1 & 1.1 \\
$d \times 10^{6}, \mathrm{~m}$ & 1.2 & 1.3 \\
\hline
\end{tabular}

the straight lines to a satisfactory extent, thus confirming the validity of the model established.

In order to determine the temperature at the reaction front that is of significant interest, the parameters pertaining to the resistance terms were extracted from reliable sources. ${ }^{10,11)}$ Given the expression of reaction rate constant adopted by García-Labiano et al., ${ }^{12)}$ the front temperature can be calculated based on $\delta_{2}$. After that, an average lime layer porosity of 0.45 that lies in the range summarized by Stanmore and Gilot, ${ }^{13)}$ is assumed as a first approximation. The effective thermal conductivity and grain size can therefore be obtained. The calculated results are listed in Table 2, where the front temperature is generally much higher than $1173 \mathrm{~K}$ and becomes higher as the bulk liquid temperature increases. The thermal conductivity appears to be independent of temperature and the calculated values correspond well to the ones given by Hills, ${ }^{14)}$ who employed a similar methodology to determine the parameter in a gassolid system. The calculated grain size enlarges as the bulk temperature increases, which could be explained by the enhancement of sintering at a high temperature. In addition, the calculated sizes agree well with the ones reported by Borgwardt. ${ }^{15)}$

\section{Brief Summary and Future Prospects}

A kinetic model for limestone decomposition under BOF steelmaking condition has been built on the basis of shrinking core hypothesis. The model has been demonstrated to be a useful tool for determining critical parameters using experimentally measured data. Nonetheless, a large number of issues still remain unexplored including the questions of how to express structural variations of lime layer during calcination and how slagging proceeds as limestone decomposes in a converter. Here, both experimental work in laboratory and industrial trials will be undertaken. Attempts will also be made to determine the controlling step of the decomposition process both in hot metal and molten slag by using the present model.

\section{REFERENCES}

1) J. Pal, S. Ghorai, D. P. Singh, A. K. Upadhyay, S. Ghosh, D. Ghosh and D. Bandyopadhyay: ISIJ Int., 50 (2010), 105.

2) T. Deng, P. Nortier, M. Ek and S. Du: Metall. Trans. B, 44 (2013), 98.

3) A. P. Watkinson and J. K. Brimacombe: Metall. Trans. B, 13 (1982), 369.

4) S. Mukawa and Y. Mizukami: ISIJ Int., 35 (1995), 1374.

5) Y. Wei, Y. Wang and N. Li: Ironmaking Steelmaking, 41 (2014), 229.

6) J. Szekely, J. W. Evans and H. Y. Sohn: Gas-Solid Reactions, Academic Press, New York, (1976), 66.

7) O. Levenspiel: Chemical Reaction Engineering, John Wiley \& Sons, New York, (1999), 570.

8) G. Sun: Master's Thesis, Northeastern University, Shenyang, (2015), 42.

9) G. D. Silcox, J. C. Kramlich and D. W. Pershing: Ind. Eng. Chem. Res., 28 (1989), 155.

10) A. Bes: Doctoral Thesis, Otto von Guericke University of Magdeburg, Magdeburg, (2006), 44.

11) M. El-Fakharany: Doctoral Thesis, Otto von Guericke University of Magdeburg, Magdeburg, (2012), 60.

12) F. García-Labiano, A. Abad, L. F. Diego, P. Gayán and J. Adánez: Chem. Eng. Sci., 57 (2002), 2381.

13) B. R. Stanmore and P. Gilot: Fuel Process Technol., 86 (2005), 1707.

14) A. W. D. Hills: Chem. Eng. Sci., 23 (1968), 297.

15) R. H. Borgwardt: Chem. Eng. Sci., 44 (1989), 55. 